\title{
Analysis of transmission characteristics of non- line-of-sight ultraviolet light under complex channel conditions
}

\author{
Xuan Zheng ${ }^{1}$, Yanfeng Tang ${ }^{1, *}$, and Jingyi $\mathrm{Du}^{1}$ \\ ${ }^{1}$ Changchun university of science and technology, Weixing road 7089, Changchun, JILIN province, \\ China
}

Keywords: Ultraviolet light communication, Non-line-of-sight, Nonspherical particles, Spherical particles, Scattering coefficient.

\begin{abstract}
Using the multiple scattering model of non-line-of-sight ultraviolet light to simulate and analyze the atmospheric channel characteristics in the complex environment of haze and dust. The Mie scattering theory and T matrix method are used to analyze the path loss of spherical particles and non-spherical particles with particle concentration at different communication distances. The results show that when the communication distance is less than 50 meters, the communication quality under severe haze is the best, and for long-distance communication, the path loss under severe haze increases almost proportionally. In the nonline-of-sight ultraviolet light communication link, the higher the concentration of dust particles, the better the communication quality of the non-line-of-sight ultraviolet light communication transmission. Analysis of the scattering coefficient of spherical particles is significantly greater than that of non-spherical particles.
\end{abstract}

\section{Introduction}

Ultraviolet communication uses ultraviolet light in the "solar blind zone" as the c arrier wave and the free space of the atmosphere as the channel to transmit signals ${ }^{[1]}$. Ultraviolet light communication has a non-line-of-sight link, using the unique scattering characteristics of ultraviolet light, without the need for precise alignment of the receiving and transmitting ends, so as to complete the signal transmission ${ }^{[2]}$. The quality of UV communication is affected by the shape and concentration of aerosol molecules in the atmosphere. Therefore, it is very important to study the transmission characteristics of ultraviolet light in a complex channel environment. In this paper, miesian scattering theory and T-matrix theory are used to analyze the transmission characteristics of ultraviolet light under different concentrations of haze and dust, and a multi-scatter link model of ultraviolet NLOS link is established. The paper studies the path loss of uv light scattering under different haze and dust concentrations, and simulates the influence of different particle morphology on the multiple

\footnotetext{
* Corresponding author: tangyanfeng@cust.edu.cn
} 
scattering of UV light under different channel conditions, thus providing a theoretical basis for the design and optimization of NLOS UV communication system under different channel environments.

\section{Theoretical basis}

\subsection{Scattering theory of atmospheric particles}

Aerosol refers to multiple dispersions composed of various particles in the atmosphere, and the aerosol with a particle size of $0.01-1 \mu \mathrm{m}$ is called haze ${ }^{[3]}$. When the particle size of the spherical particle increases to a certain point $x=2 \pi a / \lambda>0.1-0.3$ (a is the particle radius and $\lambda$ is the wavelength of the incident light), Mie scattering can be used to calculate the scattering coefficient of the spherical particle.

$a_{n}$ and $b_{n}$ represent the Mie scattering coefficient, and $\tau_{n}$ and $\pi_{n}$ represent the angular scattering coefficient, which are related to the scattering Angle.The expressions of $a_{n}$ and $b_{n}$ are as follows ${ }^{[4]}$ :

$$
\begin{aligned}
& a_{n}=\frac{\varphi_{n}(\alpha) \varphi_{n}(m \alpha)-m \varphi_{n}(\alpha) \varphi_{n}(m \alpha)}{\xi_{n}(\alpha) \varphi_{n}(m \alpha)-m \xi_{n}(\alpha) \varphi_{n}(m \alpha)} \\
& b_{n}=\frac{m \varphi_{n}(\alpha) \varphi_{n}(m \alpha)-\varphi_{n}(\alpha) \varphi_{n}(m \alpha)}{m \xi_{n}(\alpha) \varphi_{n}(m \alpha)-\xi_{n}(\alpha) \varphi_{n}(m \alpha)}
\end{aligned}
$$

According to the scattering coefficient $a_{n}$ and $b_{n}$, the scattering coefficient $K_{s}$ and absorption coefficient of a single particle $K_{a}$ are as follows:

$$
\begin{gathered}
K_{e}=\frac{2}{x^{2}} \sum_{n=1}^{\infty}(2 n+1)\left\{\operatorname{Re}\left(a_{n}+b_{n}\right)\right\} \\
K_{\mathrm{a}}=\frac{2}{x^{2}} \sum_{n=1}^{\infty}(2 n+1)\left(\left|a_{n}\right|^{2}+\left|b_{n}\right|^{2}\right)
\end{gathered}
$$

Aerosol molecules in atmospheric free channel are not only complex in composition, but also have different shapes. Although spherical particles are calculated with high precision by Using The Mie scattering theory, most particles in the atmosphere are not uniformly spherical.In this paper, T matrix is used to solve the extinction of non-spherical particles. The rotating body of the $\mathrm{T}$ matrix method uses the electric current generated on the surface of the scattering particle to replace the scattering internal field of the particle. Integrating the current on the particle surface is equivalent to zero internal field of the particle ${ }^{[5]}$.

For non-spherical particles with rotational symmetry, the area of the scattering cross section and the area of the extinction cross section can be expressed as ${ }^{[6]}$ :

$$
C_{s c a}=\frac{2 \pi}{k_{1}^{2}} \sum_{n=1}^{\infty} \sum_{m=-n}^{n} \sum_{n^{\prime}=1}^{\infty} \sum_{m^{\prime}=-n^{\prime}}^{n^{\prime}}\left(\left|T_{m n n^{\prime} n^{\prime}}^{12}\right|^{2}+\left|T_{m n m^{\prime} n^{\prime}}^{12}\right|^{2}+\left|T_{m n m^{\prime} n^{\prime}}^{21}\right|^{2}+\left|T_{m n m^{\prime} n^{\prime}}^{22}\right|^{2}\right)
$$




$$
C_{e x t}=-\frac{2 \pi}{k_{1}^{2}} \operatorname{Re} \sum_{n=1}^{\infty} \sum_{m=-n}^{n}\left(\left|T_{m n m^{\prime} n^{\prime}}^{11}\right|^{2}+\left|T_{m u m^{\prime} n^{\prime}}^{22}\right|^{2}\right)
$$

Where, $k_{1}$ is the wave number of the external region, $k_{1}=\omega \sqrt{\varepsilon_{1} \mu_{0}}$.

Therefore, the scattering and extinction coefficients are ${ }^{[7]}$ :

$$
Q_{\text {ext }}=C_{\text {ext }} / G, Q_{s c a}=C_{s c a} / G
$$

Where, $G$ is the projected area of the particle and the incident light in the vertical direction.

\subsection{Multi-scattering model of ultraviolet light based on Monte Carlo}

Assuming that the scattering effect of particles in the atmosphere on ultraviolet light is random, the transmitter of the system sends a large number of photons to simulate the transmission process of each photon. Firstly, the photon is emitted at the origin, and the propagation direction and random step length of the photon are simulated. After the photon moves, the scattering is carried out at the first scattering point $S_{1}$, and the probability that the photon is received at the receiving end at the scattering point $S_{1}$ is calculated. The photon continues to move in the new direction and stops when the set maximum scattering point or the photon survival probability is small. The photon ends its movement and is discarded. The above process assumes that each photon is independent when transmitted in the atmosphere channel, then the scattering effect of all photons emitted is the superposition of each photon scattering effect.

When a photon propagation in the atmosphere, will happen at the same time the ultraviolet light absorption and scattering effect on the atmosphere, thus the model adopts the extinction coefficient $k_{e}$ and random number of $\xi_{s}$ sampling photons moving random step length, the absorption and scattering effect of atmospheric channel affects the random step length, and the size of the weight is determined by random step, that is to say, the scattering effect not only influence the weight of the photons, also decided to the direction of propagation of photons, and absorption by changes in the weight only.

It is determined that when the photon reaches the receiving end $\mathrm{R}, \mathrm{S}_{\mathrm{n}}$ must be within the range of the receiving end cone and the movement direction of the photon is directed to the receiving end, and then the space distance method is used to determine that the transmission distance of the photon is within the receiving cone range.The spatial distance determination is shown in the figure below. When the photon reaches the $n$th scattering point $S_{n}$, the photon coordinate is at this time. When determining whether the next scattering point $S_{n+1}$ coordinate of the photon reaches the receiving surface, two cases are considered: (1) If the photon is scattered If the route $S_{n} S_{n+1}$ is parallel to the receiving surface, the photon is not received and reaches the scattering point $S_{n+1}$ to continue scattering. (2) If the photon scattering route $S_{n} S_{n+1}$ intersects the receiving surface, the intersection point is $A$. If the distance from the center of $\mathrm{A}$ to the receiving surface is less than the radius of the receiving surface, the photon is received by the receiving end.

After multiple scattering, the photon reaches the receiving end cone and needs to meet three conditions: (1)The photon can move distance, and $l \geq \mathrm{AN}$ (2) The moving direction of the photon is within the range of the receiving surface cone. (3)The scattering point $S_{n}$ reaches the receiving cone of the receiving end $\mathrm{R}$. If the scattering point reaches the receiving surface, $\zeta_{\mathrm{Sn}}<\phi_{r}$ can calculate whether the photon is received. 


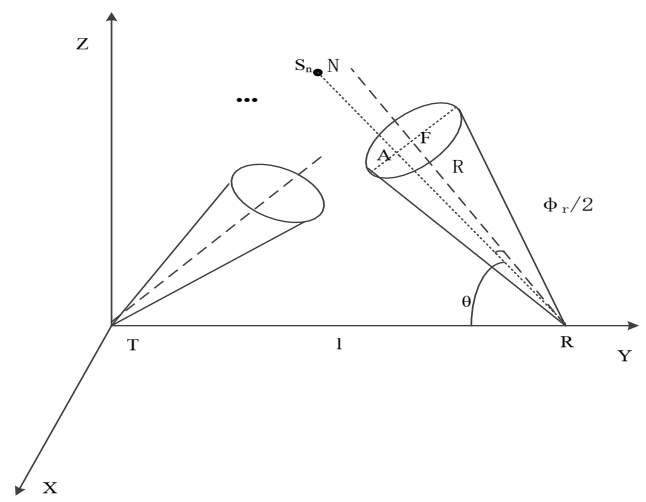

Fig. 1-1. Spatial distance method to determine the photon receiving diagram.

The probability that a photon is directly received without scattering $\mathrm{P}_{0}=0$. The weight of the photon decreases after multiple scattering, so the number of photon scattering is set as $\mathrm{N}$. In the transmission process of photon, each scattering occurs as an irrelevant event, and the probability of photon scattering $\mathrm{N}$ times arrives at the receiving end and is received is:

$$
\mathrm{P}_{\mathrm{N}}=\sum_{n=1}^{N} \mathrm{P}_{\mathrm{n}}
$$

\section{Simulation and analysis}

Path loss ${ }^{[8]}$ describes the reduction of optical power in the process of UV communication due to path attenuation. The more path loss in photon transmission, the worse communication quality. Suppose the emitter emits M photons, and the probability that the $\mathrm{M}$ photon reaches the receiving end through N-times scattering is Pmn, then the probability that the emitter emits a photon to reach the receiving end and be received is ${ }^{[9]}$ :

$$
P=\frac{\sum_{m=1}^{M} P_{m n}}{M}
$$

Then the path loss of non-line-of-sight ULTRAVIOLET communication is:

$$
P L=10 \lg \frac{1}{P}
$$

\subsection{Scattering channel analysis of spherical particles}

The Mie scattering theory was used to analyze the path loss of haze spherical particles with different concentrations in non-line-to-view UV communication. The wavelength of UV light was $265 \mathrm{~nm}$, the complex refractive index was $m=1.52+0.005 i$, the radius of the particles was $0.1 \mathrm{~m}$, the transmitter-receiver elevation Angle was $\theta_{t}=\theta_{r}=20^{\circ}$ the asymmetric factor was $g=0.72$, the emitted $\mathrm{m}=10^{7}$ photons, and the maximum scattering 
times was $\mathrm{N}=5$.

Analyze the path loss of different concentrations of spherical sand dust particles in nonline-of-sight ultraviolet communication. Using Mie's scattering theory, using ultraviolet light with a wavelength of $266 \mathrm{~nm}$, the complex refractive index of the dust particles is $m=1.07+0.688 i$, and the particle size of the sand dust is $10 \mathrm{~nm}$. The simulation parameters are the same as those of spherical haze particles. The results are shown in Figure 2-2 below.

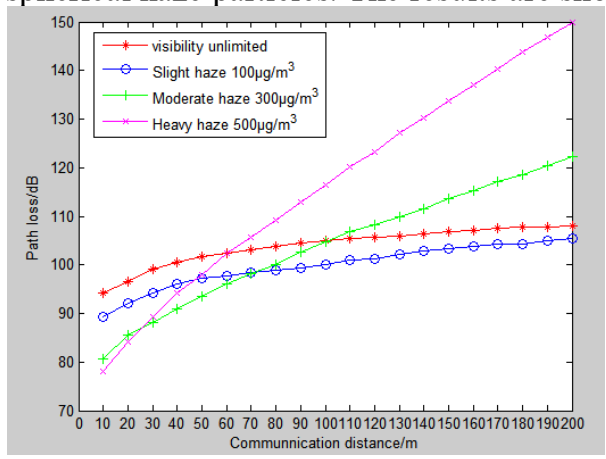

Fig. 2-1. Transmission path loss of spherical haze particles.

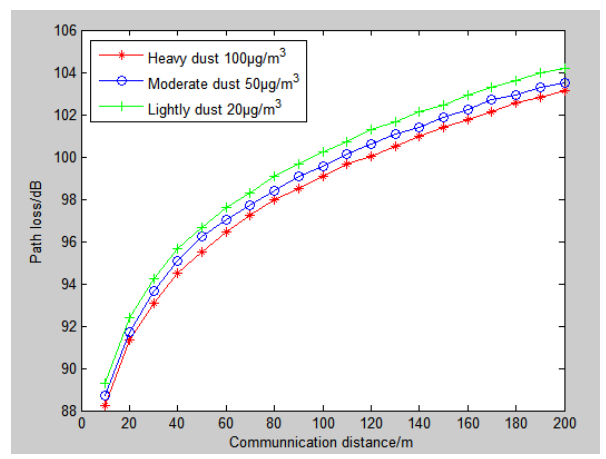

Fig. 2-2. Transmission path loss of spherical dust particles.

It can be seen from the figure that in non-line-to-sight UV communication, path loss under moderate and severe haze is about $10 \mathrm{~dB}$ lower than that under mild haze and sunny environment respectively in short distance communication. When the communication distance is $10 \mathrm{~m}$, the loss ratio of non-line-to-sight communication path in a clear day is $22 \mathrm{~dB}$ higher than the haze concentration of $500 \mathrm{~g} / \mathrm{m}^{3}$. In the long distance communication, the increase of the absorption effect makes the extinction coefficient of UV light increase. In the environment of moderate and severe haze, the communication quality under the condition of moderate and severe haze is worse than that under the condition of mild haze and fine weather, which increases the path loss.It can be seen from the figure that in nonline-of-sight ultraviolet communication, the transmission path loss of spherical sand dust particles. When the concentration of sand dust particles is low, the absorption effect of the particles is greater than the scattering effect, and a large number of photons die out, which causes the path loss to increase. When the concentration of sand dust particles increases, the scattering effect of the particles increases, which affects non-line-of-sight ultraviolet light. Communication performance has a certain improvement effect.

\subsection{Analysis of non-spherical particle scattering channel}

The rotating symmetric ellipsoid haze particles andnon-spherical dust particles with an 
axial ratio of 2 were studied, and the simulation parameters were the same as the spherical haze particles. The path losses of non-line-of-sight UV light transmission under different concentrations of non-spherical haze particles and non-spherical dust particles are studied, and the results are shown in Figure 2-3, Figure 2-4.

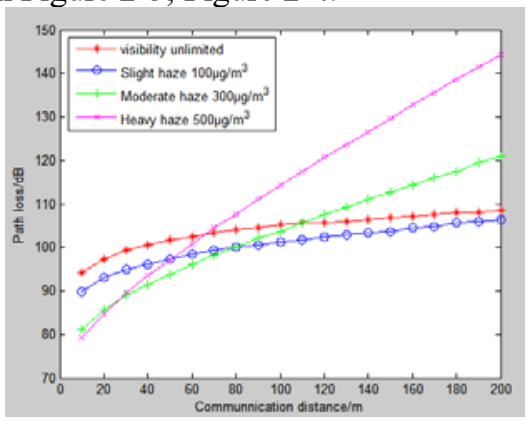

Fig. 2-3. Transmission path loss of non-spherical haze particles.

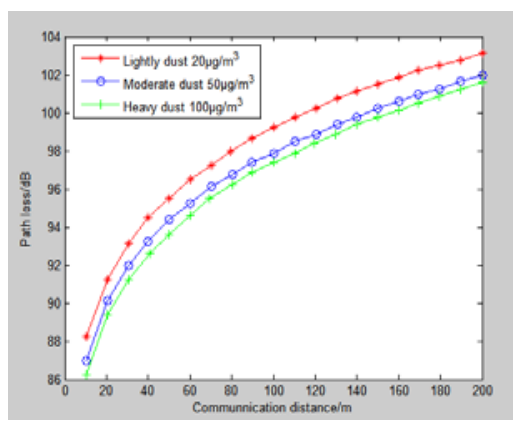

Fig. 2-4. Transmission path loss of non-spherical dust particles.

It can be seen from Figure 2-3 that in the case of short-distance communication, under severe haze conditions, the communication quality is the best and the path loss is the smallest. Comparing Figure 2-1, we can see that the communication quality of nonspherical haze particles is better than that of spherical particles at the same particle concentration. The scattering cross section of spherical particles increases the attenuation coefficient of ultraviolet light and reduces the communication quality.It can be seen from the figure that as the communication distance increases, the path loss of non-line-of-sight ultraviolet communication without the non-spherical dust particle concentration also gradually increases. Comparing Figure 2-2, we can see that the communication quality of ellipsoidal dust particles is better than that of spherical particles under the same particle concentration. The scattering cross-section of ellipsoidal dust particles is smaller than that of spherical dust particles, and the attenuation coefficient is small, and the path loss is small.

\section{Conclusion}

By using Mie scattering theory and T matrix method, the multi-scattering model of nonline-of-sight link UV light is established, and combined with the size distribution function of haze particles and dust particles, the path loss of multi-transmission of non-line-of-sight UV light communication under different concentrations is analyzed. The results show that: 1) When the communication distance is less than 50 meters, the path loss of non-line-tosight UV light transmission is the smallest in the case of heavy haze, and the communication quality can be effectively improved by increasing the concentration of fog haze particles. Appropriately increasing the concentration of haze particles can effectively 
improve the communication quality; as the communication distance increases The path loss of non-line-of-sight ultraviolet light increases almost proportionally with the increase of the haze particle concentration, and the communication quality drops sharply. When the dust particle size and the communication distance are the same, the communication quality of the non-line-of-sight ultraviolet communication will improve with the increase of the dust concentration, and the path loss will decrease. 2) In the case of non-spherical particles, the path loss of non-line-of-sight UV communication is less than that of spherical particles, and the scattering coefficient is increased due to the large scattering cross section of spherical particles. In the actual situation, the single scattering of non-line-of-sight ultraviolet light communication is very small, and the particles in the atmosphere are not all spherical particles. Therefore, it is very necessary to study the channel characteristics of multiple scattering of non-line-of-sight ultraviolet light communication between spherical and nonspherical particles.

\section{References}

1. Wang G, Wang K, Gong C, et al. A 1Mbps Real-time NLOS UV Scattering Communication System with Receiver Diversity over $1 \mathrm{~km}[\mathrm{~J}]$. IEEE Photonics Journal, 2018, 10(2):1-13.

2. Ding $\mathrm{Y}$, Tong S. A simplified NLOS UV communication model[C]//International Conference on Optoelectronics \& Microelectronics. IEEE, 2012.

3. Xingna $\mathrm{Yu}, \mathrm{Ma} \mathrm{J}$, An $\mathrm{J}$, et al. Impacts of meteorological condition and aerosol chemical compositions on visibility impairment in Nanjing, China[J]. Journal of Cleaner Production, 2016.

4. Peng Song, Jiefeng Wan, Chong Chen, Taifei Zhao, Yunhong Li. The influence of haze weather on ultraviolet communication[J]. Acta Photonica Sinica, 2018, 47(05): 143-152.

5. Xingna Yu, Ma J, An J, et al. Impacts of meteorological condition and aerosol chemical compositions on visibility impairment in Nanjing, China[J]. Journal of Cleaner Production, 2016.

6. Qiang Xu, Dongqin Wang, Xu Wang, Zhensen Wu. Calculation and analysis of the scattering characteristics of simple non-spherical particles of atmospheric haze by using the T matrix method[J]. Infrared and Laser Engineering, 2017, 46(11): 207-213.

7. Bijun Hu. Simulation research on polarization transmission characteristics of nonspherical particles based on $\mathrm{T}$ matrix[D]. Changchun University of Science and Technology, 2018.

8. Jianyu Wang. Research on the performance of non-direct-view ultraviolet communication under atmospheric turbulence and haze [D]. Xi'an Polytechnic University, 2017.

9. Yangyu Xiong, Peng Song, Jianyu Wang, Fei Song. Node design and performance analysis of ultraviolet communication network[J]. Journal of Xi'an Engineering University, 2016, 30(06): 797-801. 